ANNALS OF

CLINICAL

NEUROPHYSIOLOGY

\title{
Investigation of serum biomarkers for neuropathic pain in neuromyelitis optica spectrum disorder: a preliminary study
}

\author{
Jae-Won Hyun', Yeseul Kim² ${ }^{2}$ Ho Jin Kim,2 \\ 'Department of Neurology, Division of Clinical Research, National Cancer Center, Goyang, Korea \\ ${ }^{2}$ Research Institute and Hospital of National Cancer Center, Goyang, Korea
}

Received: October 27, 2020

Revised: December 23, 2020

Accepted: February 25, 2021

\section{Correspondence to}

\section{Jae-Won Hyun}

Department of Neurology, Division of Clinical Research, National Cancer Center, 323 Ilsan-ro, Ilsandong-gu, Goyang 10408, Korea

Tel: +82-31-920-1660

Fax: +82-31-905-5524

E-mail:12141@ncc.re.kr

ORCID

\section{Jae-Won Hyun}

https://orcid.org/0000-0003-0288-771X Yeseul Kim

https://orcid.org/0000-0003-0738-5508

Ho Jin Kim

https://orcid.org/0000-0002-8672-8419

Background: We aimed to investigate candidates for serological biomarkers of neuropathic pain in individuals with neuromyelitis optica spectrum disorder (NMOSD).

Methods: We analyzed 38 sera samples from 38 participants with NMOSD in National Cancer Center. Neuropathic pain was evaluated using the painDETECT questionnaire. Pain with neuropathic components (painDETECT score $\geq 13$ ) was observed in 22 participants, among whom 17 had definite neuropathic pain (painDETECT score $\geq 19$ ). The remaining 16 participants had non-neuropathic pain (painDETECT score $<13$ ). Serum glial fibrillary acidic protein (GFAP) levels were assessed using a single-molecule array assay. Several cytokines, including tumor necrosis factor-alpha (TNF-a), interleukin (IL)-6, IL-10, and IL-17A, were measured by a multiplex bead-based immunoassay.

Results: In comparison of NMOSD participants with neuropathic pain components (or definite neuropathic pain) and those with non-neuropathic pain, the absolute values of serum GFAP, TNF-a, IL-6, and IL-10 levels were higher in participants with neuropathic pain components (or definite neuropathic pain), but these findings did not reach statistical significance. Conclusions: Further larger-scale investigations to find reliable serological biomarkers for neuropathic pain in NMOSD are warranted.

Key words: Neuromyelitis optica; Neuralgia; Biomarker; Serum

This is an Open Access article distributed under the terms of the Creative Commons Attribution Non-Commercial License (http:// creativecommons.org/licenses/by-nc/4.0) which permits unrestricted non-commercial use, distribution, and reproduction in any medium, provided the original work is properly cited. 


\section{INTRODUCTION}

Neuromyelitis optica spectrum disorder (NMOSD), an inflammatory disease of the central nervous system (CNS), is associated with neuropathic pain, which arises as a direct consequence of inflammatory lesions in the somatosensory nervous system. ${ }^{1,2}$ Neuropathic pain can be usually resistant to therapeutic approaches, but there is no standardized objective quantitative biomarker for neuropathic pain in individuals with NMOSD. Identifying such biomarkers may be helpful for monitoring neuropathic pain, which can affect the quality of life of these patients in clinical practice. ${ }^{3}$

Cytoskeletal proteins of astrocytes (like glial fibrillary acidic protein [GFAP]) could be candidates for biomarkers of neuropathic pain since the pathophysiology of NMOSD is primarily astrocytopathy. ${ }^{4}$ Because NMOSD is an inflammatory condition of the CNS, cytokines involved in the inflammatory process could also be candidates for biomarkers of neuropathic pain. ${ }^{5}$ In this preliminary study, we aimed to evaluate potential serological biomarkers of neuropathic pain in individuals with NMOSD.

\section{MATERIALS AND METHODS}

A total of 38 sera samples from 38 individuals with NMOSD who visited the National Cancer Center (NCC) were ana- lyzed. The Korean version of the painDETECT questionnaire was investigated as previously described at remission status (after at least 3 months of relapses)., 3 Participants with available stored serum samples, collected at remission periods were randomly enrolled; 26 were simultaneously obtained with questionnaire and 12 were obtained after administering questionnaire but within 3 month-interval of sampling and administering questionnaire. ${ }^{3,6}$ All samples were stored at $-80^{\circ} \mathrm{C}$ prior to analysis. All participants satisfied the 2015 diagnostic criteria for NMOSD and were positive for AQP4 antibodies tested by in-house live cell-based assays. ${ }^{7,8}$ PainDETECT scores ranged from 0 to 38, and pain is classified 3 categories; 19-38: strongly suggestive of neuropathic pain (definite neuropathic pain), 13-18: suggestive of pain with neuropathic components, and < 13: suggestive of non-neuropathic pain. ${ }^{6}$ Among the 38 participants with NMOSD, 22 had experienced pain with neuropathic components assessed by painDETECT (score $\geq 13$ ), but 16 had non-neuropathic pain (score $<13)$. Additionally, 17 participants had experienced definite neuropathic pain (score $\geq 19$ ), and these participants were also compared to 16 participants with non-neuropathic pain. This study was approved by the Institutional Review Board of the NCC.

Several candidate biomarkers of neuropathic pain in NMOSD participants, including GFAP and cytokines (tumor necrosis factor-alpha [TNF-a], interleukin [IL]-6, IL-10, IL-17A) were investigated. GFAP levels were assessed using a com-

\section{Table 1. Demographics}

\begin{tabular}{|c|c|c|c|}
\hline & Pain with neuropathic component $(n=22)$ & Non-neuropathic pain $(n=16)$ & $p$-value \\
\hline Age (years) & $46.5 \pm 6.0$ & $42.8 \pm 10.5$ & 0.246 \\
\hline Sex (female to male ratio) & $20: 2$ & $16: 0$ & 0.215 \\
\hline EDSS (median) & 3.5 & 3.0 & 0.100 \\
\hline Disease duration & $10.3 \pm 5.3$ & $11.0 \pm 6.4$ & 0.722 \\
\hline \multirow[t]{2}{*}{ Presence of prior spinal cord attack } & $22 / 22$ & $16 / 16$ & - \\
\hline & Definite neuropathic pain $(n=17)$ & Non-neuropathic pain $(n=16)$ & $p$-value \\
\hline Age (years) & $45.6 \pm 6.0$ & $42.8 \pm 10.5$ & 0.402 \\
\hline Sex (female to male ratio) & $15: 2$ & $16: 0$ & 0.485 \\
\hline EDSS (median) & 3.5 & 3.0 & 0.108 \\
\hline Disease duration & $10.5 \pm 5.6$ & $11.0 \pm 6.4$ & 0.823 \\
\hline Presence of prior spinal cord attack & $17 / 17$ & $16 / 16$ & - \\
\hline
\end{tabular}

Values are presented as mean \pm standard deviation or number.

EDSS, expanded disability status scale. 
mercial single-molecule array assay (Simoa, Quanterix Corp., Billerica, MA, USA). Cytokines were measured by a multiplex bead-based immunoassay (cytometric bead array) according to the manufacturer's protocol (BD Bioscience, San Jose,
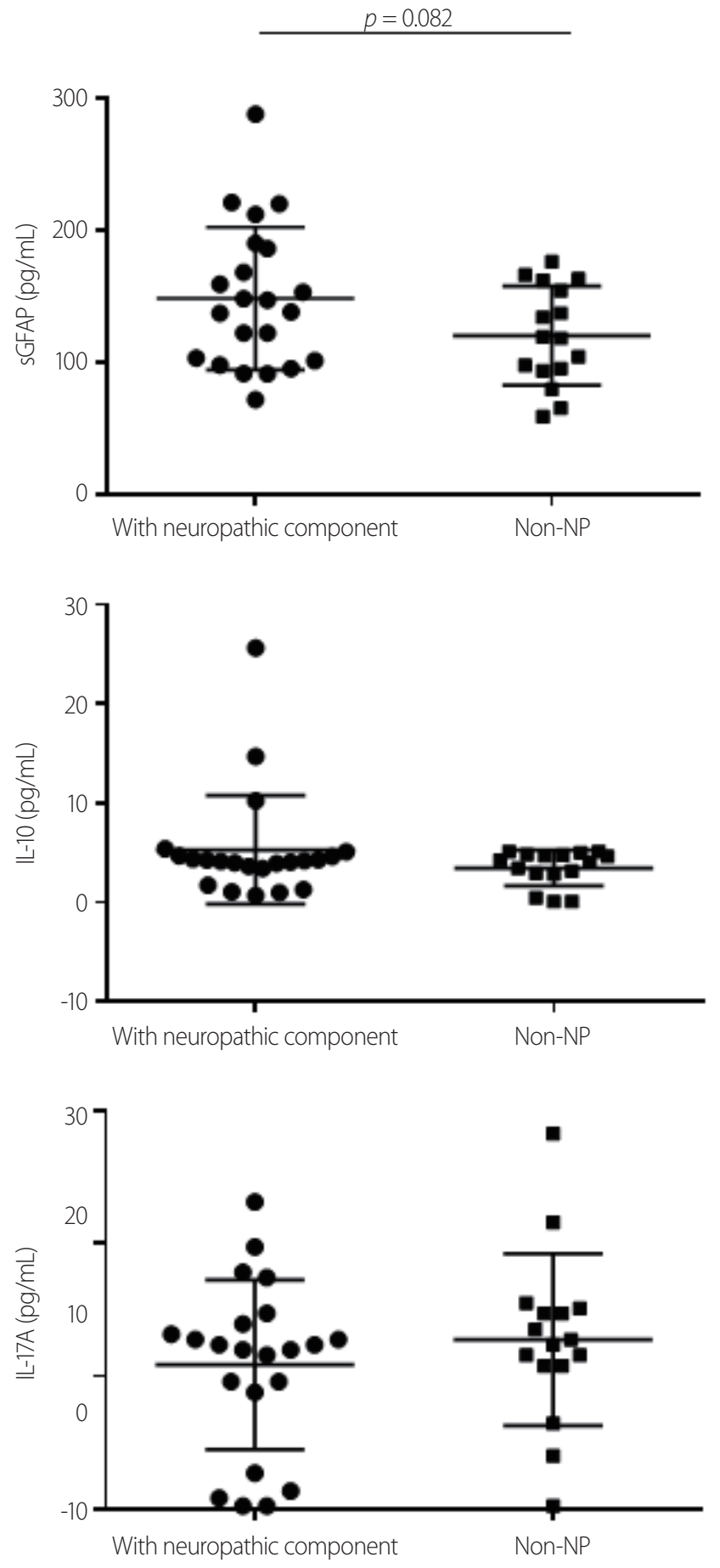

(A, USA). GFAP and cytokine levels were independently examined by a blinded investigator. The Student's $t$-test and Mann-Whitney test were used to compare the levels of serum biomarkers.
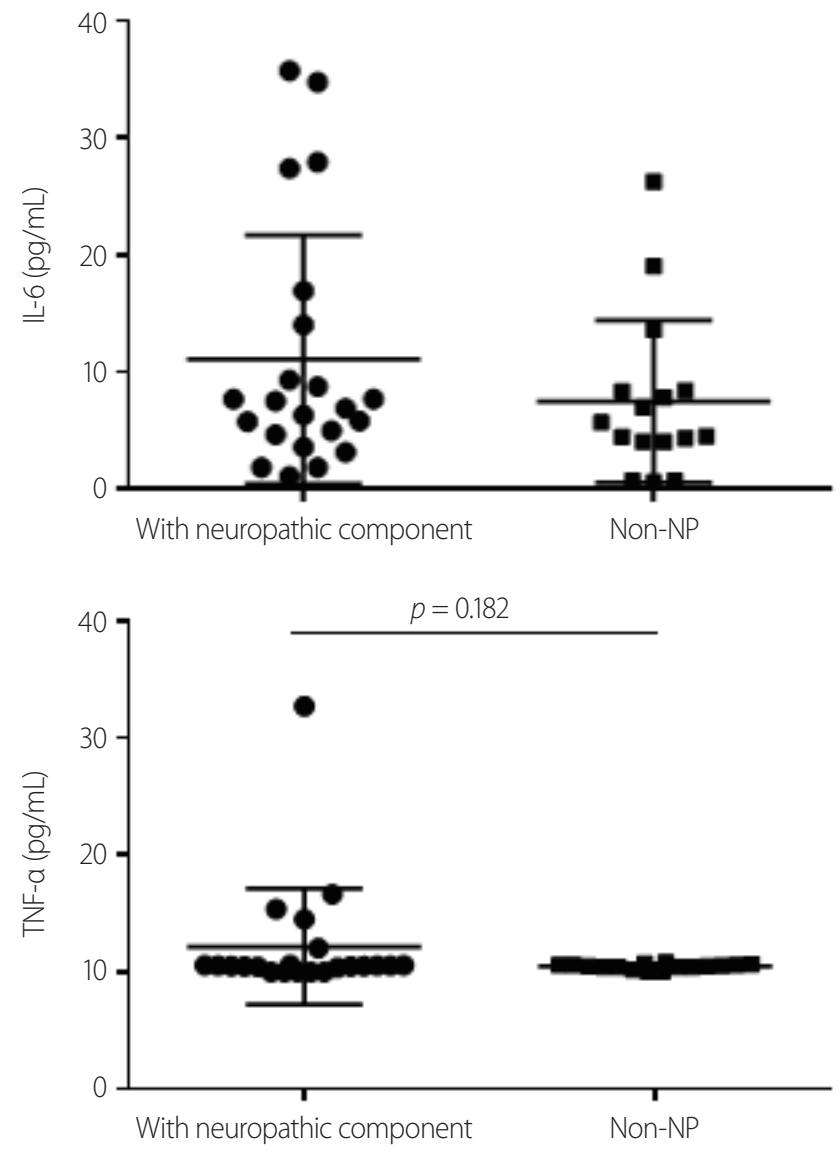

Fig. 1. Comparison of levels of serum biomarkers between neuromyelitis optica spectrum disorder participants with neuropathic pain component and non-neuropathic pain (NP). GFAP, glial fibrillary acidic protein; IL, interleukin; TNF-a, tumor necrosis factor-alpha. 


\section{RESULTS}

\section{Demographics of participants}

Table 1 shows the demographics of participants with NMOSD. The female-to-male ratio and age at sampling of 22 participants with (score $\geq 13$ ) and 16 participants without (score < 13) neuropathic pain components (non-neuropathic pain) were $20: 2$ and 16:0, and $46.5 \pm 6.0$ and $42.8 \pm 10.5$ years, respectively. The disease duration of NMOSD participants with and without neuropathic pain components were $10.3 \pm 5.3$ and $11.0 \pm 6.4$ years, respectively. The median current Expanded Disability Status Scale values for participants with and without neuropathic pain components were 3.5 and 3.0, respectively. Additionally, the demographics of 17 participants who had definite neuropathic pain (score $\geq 19$ ) were also compared with those of 16 participants who had non-neuropathic pain. There were no statistically significant differences in demographics between the two groups.

\section{Levels of serum biomarkers in NMOSD participants with neuropathic pain components (or definite neuropathic pain) and non-neuropathic pain}

Fig. 1 and Table 2 show a comparison of the levels of individual serum biomarkers between NMOSD participants with and without neuropathic pain components (non-neuropathic pain). The absolute mean values of serum GFAP (148.3 \pm 1.5 vs. $120.1 \pm 9.4 \mathrm{pg} / \mathrm{mL}, p=0.082)$, TNF-a $(10.8 \pm 5.3$ vs. 2.4 $\pm 0.3 \mathrm{pg} / \mathrm{mL}), \mathrm{IL}-6(11.1 \pm 2.3$ vs. $7.5 \pm 1.7 \mathrm{pg} / \mathrm{mL})$, and $\mathrm{IL}-10$
(5.3 \pm 1.2 vs. $3.5 \pm 0.4 \mathrm{pg} / \mathrm{mL}$ ) levels were higher in NMOSD participants with neuropathic pain components than in those without neuropathic pain components but the difference did not reach statistical significance.

Fig. 2 and Table 2 show a comparison of the levels of individual serum biomarkers between NMOSD participants with definite neuropathic pain and non-neuropathic pain. The absolute mean values of serum GFAP $(149.1 \pm 14.1$ vs. 120.1 $\pm 9.4 \mathrm{pg} / \mathrm{mL}, p=0.099), \mathrm{TNF}-\mathrm{a}(6.9 \pm 2.5 \mathrm{vs} .2 .4 \pm 0.3 \mathrm{pg} / \mathrm{mL}$, $p=0.088), \mathrm{IL}-6(10.5 \pm 2.5$ vs. $7.5 \pm 1.7 \mathrm{pg} / \mathrm{mL})$, and IL-10 (4.5 \pm 0.8 vs. $3.5 \pm 0.4 \mathrm{pg} / \mathrm{mL}$ ) levels were higher in NMOSD participants with definite neuropathic pain than in those with non-neuropathic pain but the results did not reach statistical significance.

\section{DISCUSSION}

In comparison of NMOSD participants with neuropathic pain components (or definite neuropathic pain) and those without neuropathic pain, the non-significant higher tendency of serum GFAP, TNF-a, IL-6, and IL-10 levels were observed in the group with neuropathic pain components (or definite neuropathic pain). Future larger-scale investigations of serum GFAP, TNF- $a$, IL-6, and IL-10 levels as potential biomarkers for neuropathic pain in NMOSD are required to find surrogate serological indicators.

Neuropathic pain is an important symptom in NMOSD

Table 2. Individual levels of serum biomarkers

\begin{tabular}{|c|c|c|c|}
\hline & Pain with neuropathic component $(n=22)$ & Non-neuropathic pain $(n=16)$ & $p$-value \\
\hline GFAP (pg/mL) & $148.3 \pm 11.5$ & $120.1 \pm 9.4$ & 0.082 \\
\hline IL-6 (pg/mL) & $11.1 \pm 2.3$ & $7.5 \pm 1.7$ & 0.244 \\
\hline IL-10 (pg/mL) & $5.3 \pm 1.2$ & $3.5 \pm 0.4$ & 0.211 \\
\hline TNF-a (pg/mL) & $10.8 \pm 5.3$ & $2.4 \pm 0.3$ & 0.182 \\
\hline \multirow[t]{2}{*}{ IL-17A (pg/mL) } & $10.9 \pm 1.4$ & $12.8 \pm 1.6$ & 0.377 \\
\hline & Definite neuropathic pain $(n=17)$ & Non-neuropathic pain $(n=16)$ & $p$-value \\
\hline GFAP (pg/mL) & $149.1 \pm 14.1$ & $120.1 \pm 9.4$ & 0.099 \\
\hline IL-6 (pg/mL) & $10.5 \pm 2.5$ & $7.5 \pm 1.7$ & 0.358 \\
\hline IL-10 (pg/mL) & $4.5 \pm 0.8$ & $3.5 \pm 0.4$ & 0.702 \\
\hline TNF-a (pg/mL) & $6.9 \pm 2.5$ & $2.4 \pm 0.3$ & 0.088 \\
\hline IL-17A (pg/mL) & $11.1 \pm 1.7$ & $12.8 \pm 1.6$ & 0.637 \\
\hline
\end{tabular}

GFAP, glial fibrillary acidic protein; LL, interleukin; TNF-a, tumor necrosis factor-alpha. 
and can affect the quality of life. ${ }^{3}$ PainDETECT is a useful tool for detecting neuropathic pain with qualitative (categorical), but not fully objective or quantitative information in individuals with NMOSD. ${ }^{6,9}$ GFAP is a candidate for an objective
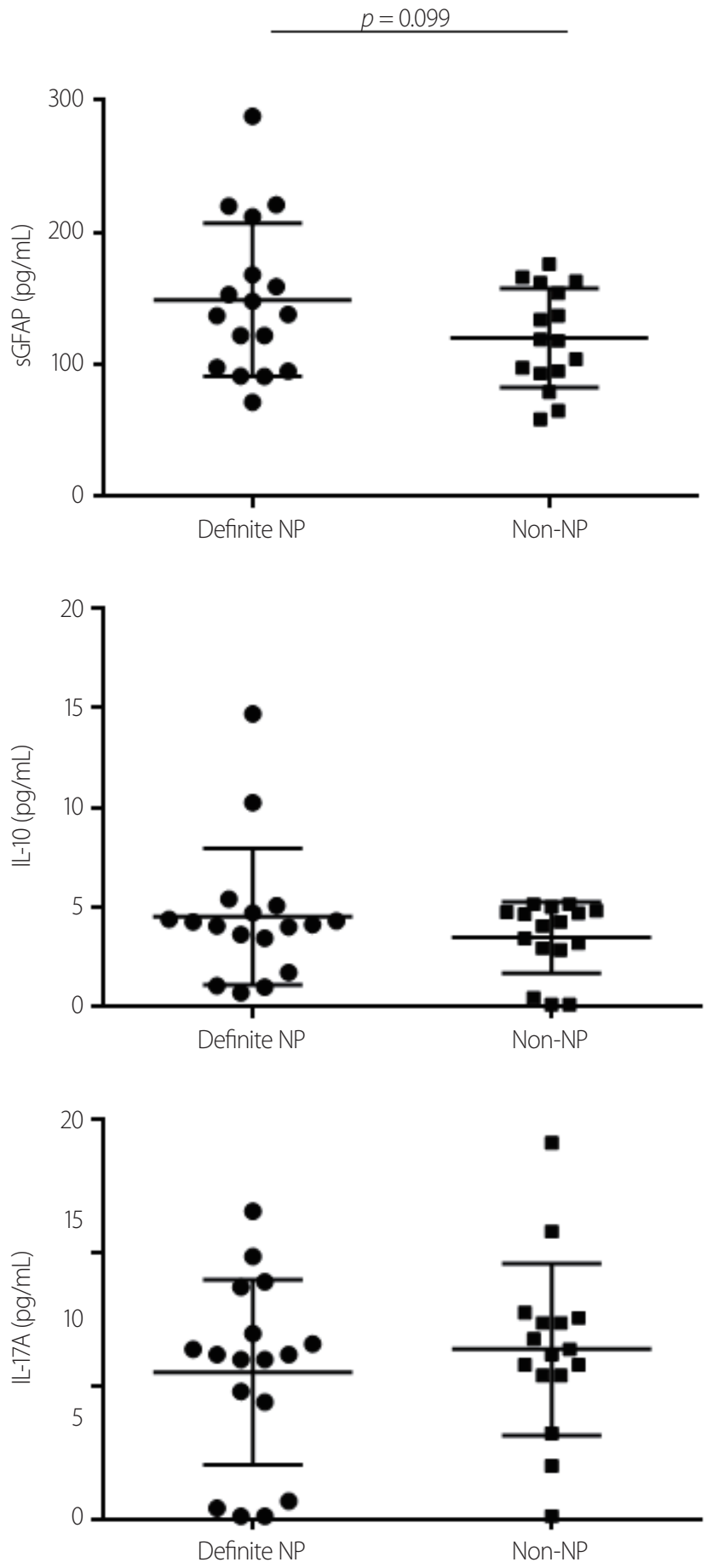

and quantitative biomarker for neuropathic pain in individuals with NMOSD because aquaporin-4 (AQP4) is mainly located in astrocytes and anti-AQP4 antibody is a pathogenic component in NMOSD..$^{10}$ Changes in GFAP, which is a
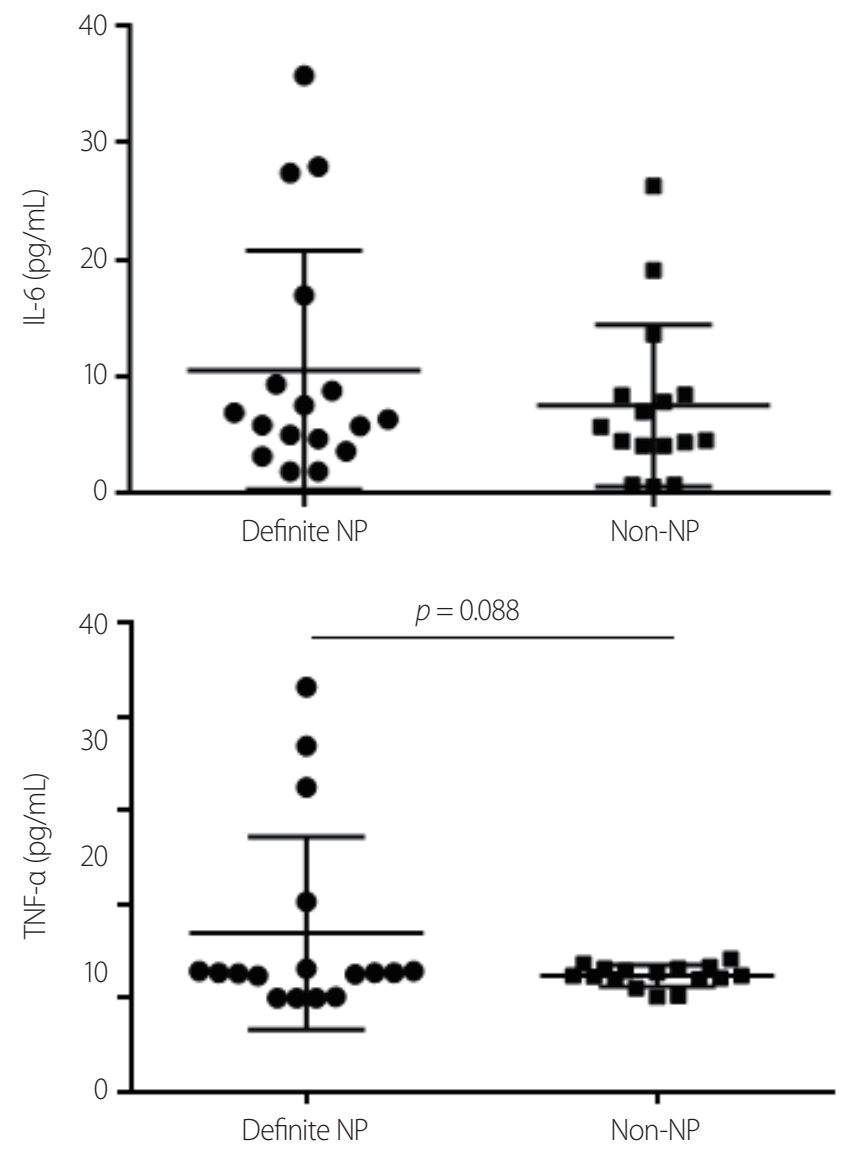

Fig. 2. Comparison of levels of serum biomarkers between neuromyelitis optica spectrum disorder participants with definite neuropathic pain (NP) and non-NP. GFAP, glial fibrillary acidic protein; IL, interleukin; TNF-a, tumor necrosis factor-alpha. 
cytoskeletal component of astrocytes, in body fluids such as serum could reflect the extent of astrocytopathy-associated inflammation which can cause neuropathic pain. ${ }^{4}$ This may explain the non-significant trend toward higher GFAP levels in NMOSD participants with neuropathic pain components (or definite neuropathic pain) than in those with non-neuropathic pain.

In the context of neuropathic pain, TNF-a is a broadly investigated inflammatory cytokine. ${ }^{11}$ In rodents, administration of exogenous TNF-a could cause allodynia, whereas a TNF-a antagonist could decrease pain. ${ }^{12,13}$ In individuals with lumbar radiculopathy, a positive correlation between low back pain disability index and TNF-a levels was observed. ${ }^{14,15}$ TNF-a inhibitors, such as etanercept and infliximab, are prescribed for painful disorders including rheumatoid arthritis, psoriatic arthritis, and ankylosing spondylitis, but the clinical efficacy of TNF-a inhibitors in neuropathic pain has shown mixed results. ${ }^{11,16}$ TNF-a inhibitors can cause CNS demyelination; ${ }^{17}$ thus TNF-a is not suitable as a therapeutic target but rather would be a candidate for a simple objective quantitative biomarker in NMOSD individuals with neuropathic pain.

Association between neuropathic pain and IL-6, IL-17A, and IL-10 levels have been demonstrated in the previous studies. ${ }^{18-23}$ IL-6 knockout mice showed less mechanical allodynia after nerve injury compared to control, and administration of an anti-IL-6 antibody conferred similar pain alleviation in rodents. ${ }^{18,19}$ In a pilot study including 7 individuals with NMOSD, treatment with a monoclonal antibody for the IL-6 receptor (tocilizumab) reduced neuropathic pain. ${ }^{20} \mathrm{Ad}$ ministration of exogenous IL-17 induced neuropathic pain, while IL-17 knockout mice showed a reduced response to pain after nerve injury. ${ }^{21,22} \mathrm{IL}-10$ elevation in the ventrolateral orbital cortex of the rat with allodynia induced by nerve injury was observed. ${ }^{23}$ However, in this study, there was no significant difference in IL-6, IL-10, or IL-17A levels between NMOSD participants with and without neuropathic pain.

The main limitations of current study are the small sample size and the possibility of unintentional selection bias, including the preferential inclusion of participants with high disease activity because of the enrollment from a single referral center. Additionally, diverse control groups and modalities to detect pain beyond painDETECT could not be included. Further larger multicenter studies with various control groups and parameters for pain measurement should be designed to establish robust serological biomarkers for neuropathic pain in NMOSD.

\section{Funding/Support}

This research was supported by a grant funded by the Korean Society of Pain and Autonomic Disorders.

\section{Financial Disclosure}

Kim Y reports no financial disclosures. Hyun has received a grant from the National Research Foundation of Korea. Kim $\mathrm{HJ}$ has lectured, consulted, and received honoraria from Alexion, Celltrion, Eisai, HanAll BioPharma, Merck Serono, Novartis, Sanofi Genzyme, Teva-Handok, and Viela Bio; received a grant from the National Research Foundation of Korea; and accepted research funding from Sanofi Genzyme, Teva-Handok, and UCB; serves on a steering committee for Medlmmune/Niela Bio; is a co-editor for the Multiple Sclerosis Journal, and an associated editor for the Journal of Clinical Neurology.

\section{Author Contributions}

Hyun and Kim HJ had full access to all of the data in the study and take responsibility for the integrity of the data and the accuracy of the data analysis. Study concept and design: Hyun, Kim HJ. Drafting of the manuscript: Hyun, Kim HJ. Acquisition, analysis and interpretation of data: All authors. Critical revision of the manuscript for important intellectual content: All authors.

\section{Conflict of Interest}

The authors have no conflicts to disclose.

\section{REFERENCES}

1. International Association for the Study of Pain (IASP). IASP terminology [Internet]. Washington, D.C. (USA): IASP; c2017 [accessed 2019 Apr 1]. Available from: www.iasp-pain.org/terminology.

2. Bradl M, Kanamori Y, Nakashima I, Misu T, Fujihara K, Lassmann H, et al. Pain in neuromyelitis optica--prevalence, pathogenesis and therapy. Nat Rev Neurol 2014;10:529-536.

3. Hyun JW, Jang H, Yu J, Park NY, Kim SH, Huh SY, et al. Comparison of neuropathic pain in neuromyelitis optica spectrum disorder and multiple sclerosis. J Clin Neurol 2020;16:124-130. 
4. Watanabe M, Nakamura Y, Michalak Z, Isobe N, Barro C, Leppert D, et al. Serum GFAP and neurofilament light as biomarkers of disease activity and disability in NMOSD. Neurology 2019;93:e1299-e1311

5. Uzawa A, Mori M, Arai K, Sato Y, Hayakawa S, Masuda S, et al. Cytokine and chemokine profiles in neuromyelitis optica: significance of interleukin-6. Mult Scler 2010;16:1443-1452.

6. Sung JK, Choi JH, Jeong J, Kim WJ, Lee DJ, Lee SC, et al. Korean version of the painDETECT questionnaire: a study for cultural adaptation and validation. Pain Pract 2017;17:494-504.

7. Wingerchuk DM, Banwell B, Bennett JL, Cabre P, Carroll W, Chitnis $T$, et al. International consensus diagnostic criteria for neuromyelitis optica spectrum disorders. Neurology 2015;85:177-189.

8. Kim Y, Kim G, Kong BS, Lee JE, Oh YM, Hyun JW, et al. Largescale in-house cell-based assay for evaluating the serostatus in patients with neuromyelitis optica spectrum disorder based on new diagnostic criteria. J Clin Neurol 2017;13:175-180.

9. Freynhagen R, Tölle TR, Gockel U, Baron R. The painDETECT project-far more than a screening tool on neuropathic pain. Curr Med Res Opin 2016;32:1033-1057.

10. Lennon VA, Wingerchuk DM, Kryzer TJ, Pittock SJ, Lucchinetti CF, Fujihara $\mathrm{K}$, et al. A serum autoantibody marker of neuromyelitis optica: distinction from multiple sclerosis. Lancet 2004;364:21062112.

11. Hung AL, Lim M, Doshi TL. Targeting cytokines for treatment of neuropathic pain. Scand J Pain 2017;17:287-293.

12. Wei XH, Zang Y, Wu CY, Xu JT, Xin WJ, Liu XG. Peri-sciatic administration of recombinant rat TNF-alpha induces mechanical allodynia via upregulation of TNF-alpha in dorsal root ganglia and in spinal dorsal horn: the role of NF-kappa B pathway. Exp Neurol 2007;205:471-484

13. Zanella JM, Burright EN, Hildebrand K, Hobot C, Cox M, Christoferson $L$, et al. Effect of etanercept, a tumor necrosis factor-alpha inhibitor, on neuropathic pain in the rat chronic constriction injury model. Spine (Phila Pa 1976) 2008;33:227-234.

14. Wang K, Bao JP, Yang S, Hong X, Liu L, Xie XH, et al. A cohort study comparing the serum levels of pro- or anti-inflammatory cytokines in patients with lumbar radicular pain and healthy subjects. Eur Spine J 2016;25:1428-1434.

15. Zu B, Pan H, Zhang XJ, Yin ZS. Serum levels of the inflammatory cytokines in patients with lumbar radicular pain due to disc herniation. Asian Spine J 2016;10:843-849.

16. US Food and Drug. Information on Tumor Necrosis Factor (TNF) Blockers (marketed as Remicade, Enbrel, Humira, Cimzia, and Simponi) [Internet]. Washington, D.C., USA: US Food and Drug; c2021 [accessed 2021 Feb 25]. Available from: https://www.fda.gov/drugs/postmarket-drug-safety-information-patients-and-providers/information-tumor-necrosis-factor-tnf-blockers-marketed-remicade-enbrel-humira-cimzia-and-simponi.

17. Kemanetzoglou E, Andreadou E. CNS demyelination with TNF-a blockers. Curr Neurol Neurosci Rep 2017;17:36.

18. Ramer MS, Murphy PG, Richardson PM, Bisby MA. Spinal nerve lesion-induced mechanoallodynia and adrenergic sprouting in sensory ganglia are attenuated in interleukin-6 knockout mice. Pain 1998;78:115-121.

19. Guptarak J, Wanchoo S, Durham-Lee J, Wu Y, Zivadinovic D, Paulucci-Holthauzen A, et al. Inhibition of IL-6 signaling: a novel therapeutic approach to treating spinal cord injury pain. Pain 2013;154:1115-1128.

20. Araki M, Matsuoka T, Miyamoto K, Kusunoki S, Okamoto T, Murata $M$, et al. Efficacy of the anti-IL-6 receptor antibody tocilizumab in neuromyelitis optica: a pilot study. Neurology 2014;82:13021306.

21. Kim CF, Moalem-Taylor G. Interleukin-17 contributes to neuroinflammation and neuropathic pain following peripheral nerve injury in mice. J Pain 2011;12:370-383.

22. Day YJ, Liou JT, Lee CM, Lin YC, Mao CC, Chou AH, et al. Lack of interleukin-17 leads to a modulated micro-environment and amelioration of mechanical hypersensitivity after peripheral nerve injury in mice. Pain 2014;155:1293-1302.

23. Shao Q, Li Y, Wang Q, Zhao J. IL-10 and IL-1 $\beta$ mediate neuropathic-pain like behavior in the ventrolateral orbital cortex. Neurochem Res 2015;40:733-739. 